\title{
PERSISTENCIA DEL CONDUCTO ARTERIAL CON HIPERTENSION PULMONAR
}

\author{
Dr. FERNANDO EIMBCKE
}

\author{
Centro Cardiovascular (Dr. Helmut Jaeger) y Cátedra de Cirugía Pediátrica (Prof. Carios \\ Urrutia). Hospital "Luis Calvo Mackenna", Santiago, Chile.
}

El desarrollo y perfeccionamiento del Cateterismo Cardíaco y su empleo rutinario como técnica auxiliar en el estudio de las Cardiopatías Congenitas, ha obligado a modificar en forma sustancial numerosos conceptos que con anterioridad se tenían especialmente acerca del pronóstico e indicación de tratamiento en determinadas Cardiopatías Congénitas. Un ejemplo lo constituye la persistencia del conducto arterial, considerada una de las cardiopatías más benignas y cuyo tratamiento era desaconsejado durante los primeros años de vida.

si bien era conocido el cuadro clínico del ductus atípico y la Dra. Taussig en su libro habla del ductus con cianosis, contraindicando su intervención; se desconocian las formas o etapas intermedias en las cuales un tratamiento oportuno podía interrumpir la evolución progresiva y fatal de una hipertensión pulmonar.

En la actualidad sabemos que el ductus atípico es debido a un aumento de la presión en el circuito pulmonar y que si bien su etiopatogenia es aún discutida conocemos su evolución, lo que nos permite formular un pronóstico y una indicación de tratamiento oportuno.

Con respecto a la frecuencia de esta complicación en el Ductus los diferentes autores dan cifras que varían entre un $10 \mathrm{y}$ un $50 \%$.

En nuestra casuística hemos logrado reunir 9 casos en un total de 34 Ductus $(25 \%)$, en un lapso de 2 años.

Queremos hacer notar que esta cifra es ligeramente inferior a la real por cuanto solo hemos incluído enfermos con diagnóstico confirmado por Cateterismo Cardíaco, excluyendo aquellos que debieron ser intervenidos sin previo cateterismo.

El aspecto clínico se analiza en el cuadro $\mathrm{N}$ ? 1 .

A pesar de lo exiguo de nuestra casuística el análisis del cuadro proyectado nos permite hacer deducciones importan-
CUADRO NQ 1

DUCTUS CON HIPERTENSION PULMONAR - 9 CABOS

\begin{tabular}{|c|c|}
\hline Sintamas & Aso: \\
\hline 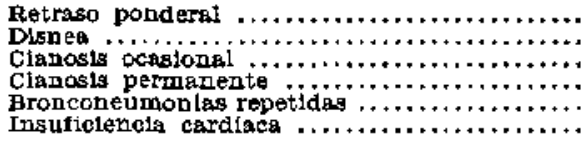 & $\begin{array}{l}7 \\
\mathbf{9} \\
\mathbf{2} \\
\mathbf{1} \\
\mathbf{7} \\
\mathbf{4}\end{array}$ \\
\hline \multicolumn{2}{|l|}{ Auscultación cerdfaca } \\
\hline 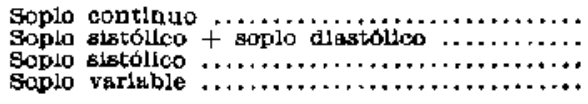 & $\begin{array}{l}3 \\
2 \\
3 \\
1\end{array}$ \\
\hline \multicolumn{2}{|l|}{ Electrocandiograma } \\
\hline 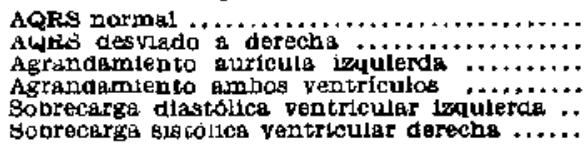 & $\begin{array}{l}8 \\
1 \\
3 \\
4 \\
4 \\
3\end{array}$ \\
\hline \multicolumn{2}{|l|}{ Radiotogia } \\
\hline 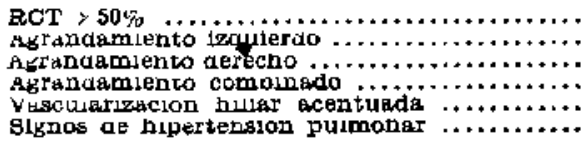 & $\begin{array}{l}7 \\
2 \\
1 \\
6 \\
9 \\
4\end{array}$ \\
\hline
\end{tabular}

tes que por lo demás concuerdan con lo senaluo por la mayoria ae los autores. Asl pouesuos ooservar que se trata de una carulopacia mal toleraua, de sintomatolugia ruausa que compromete precoz $\mathrm{y}$ grandemente el estado general de los pacuences. csto a su vez nus da la explicacion de la relativamente alta Irecuencia con que esta compucacion se senata en las casurcicas peuaricas ya que la mayoria de escos eniermos si no son tratauos precozmente tallecen en los primeros anos de vida.

un el cuadro siguiente se encuentran esquematizados los aatos hemodinamicos mas importantes en los 9 entermos estudiadios. (Cuadro No 2).

vel análsis detallado de los datos suministrados por el cateterismo es posible derivar la mayor parte de los sintomas $y$ signos auscultatorios como así mismo de los hallagos radiológicos y electrocardiográficos. Ellos dependería en últımo término de la magnitud $y$ dirección del 
CUADRO NQ 2

CATETHRIGMO CARDLAOO. 9 CABOS

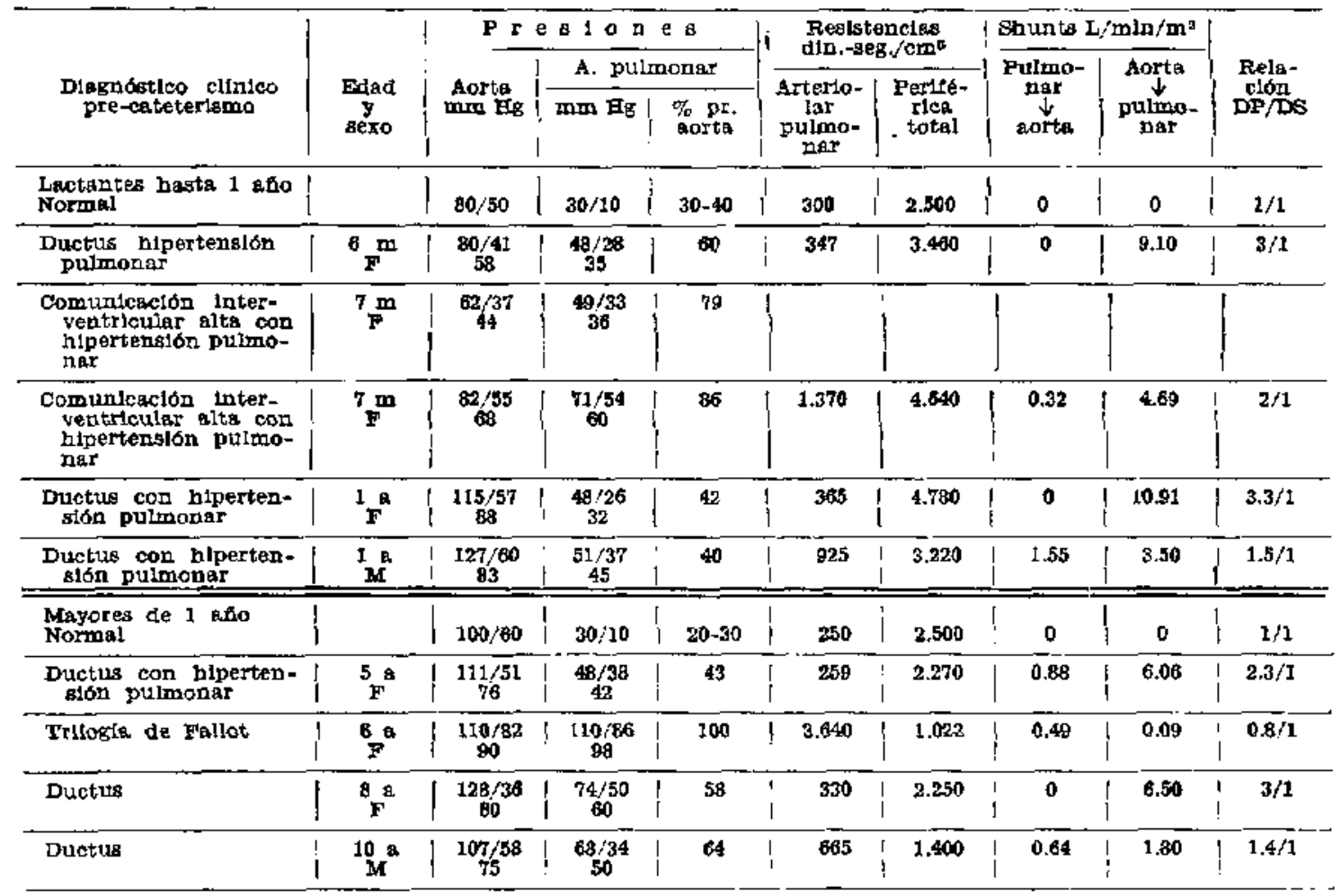

shunt a nivel del ductus, lo que a su vez está condicionado al grado de hipertensión en el circuito pulmonar y a la relación entre presión pulmonar y aórtica o sistémica.

Estas consideraciones son igualmente valederas para las demás comunicaciones entre el circuito menor $y$ el sistémico $y$ en forma especial para la comunicación interventricular alta $\mathrm{y}$ la fenestración aorto-pulmonar. Esto a su vez explica la similitud clínica entre estas malformaciones y los difíciles problemas de diagnóstico diferencial que presentan. Para corroborar esto último queremos citar lo expresado por Nadas en su libro de Cardiología Pediátrica, donde afirma que el diagnóstico diferencial del ductus atípico es uno de los problemas más difíciles y cruciales de la cardiología pediátrica y que para excluirlo debe practicarse el cateterismo cardíaco en todo enfermo con shunt izquierdo-derecho más hipertensión pulmonar y signos de hipertrofia ventricular izquierda, Si este método no logra dar una información inequívoca debe prac- ticarse la aortografía retrógrada o incluso la exploración quirúrgica.

Para terminar queremos dar cuenta del resultado del tratamiento en estos 9 enfermos. En 6 de ellos se practicó la sección del ductus con sutura de ambos cabos $y$ en 2 la oclusión se hizo por medio de ligadura. En todos ellos la evolución postoperatoria fué sin complicaciones. Queremos hacer resaltar en forma especial la mejoría clínica espectacular que se observó en los 5 lactantes, todos con un marcado retardo ponderal y 4 de ellos en insuficiencia cardíaca congestiva irreductible. Esta última se compensó fácilmente a Ios pocos días de operada y la curva de peso que había permanecido estacionaria se hizo rápidamente ascendente. Sólo en una enferma que se encontraba en la etapa que podríamos llamar final de este cuadro, no fué posible practicar la aclusión del ductus. Por ser el ejemplo tal vez más demostrativa con respecto a la evolución de esta cardiopatía queremos analizar el caso en detalle.

Se trata de una enferma de 6 años de 
edad con disnea $\mathbf{y}$ cianosis generalizada progresiva desde 1 año de edad que a la auscultación cardíaco presenta un gran reforzamiento del $2^{\circ}$ tono en la base y un saplo variable a veces dif́́cil de auscultar en el foco pulmonar.

El electrocardiograma muestra una desviación derecha del eie eléctrico de QRS $+150^{\circ}$, agrandamiento auricular derecho y signos de sobrecarga sistólica del ventrículo derecho. EI examen radiológico revela un moderado aumento de la sombra cardíaca a expensas de las cavidades derechas. Un 2 arco izauierdo muy orominente y pulsátil. Hilios marcados con hinercleridad pulmonar periférica.

Es enviada para Cateterismo Cardíaco con el diagnóstico de probable Trilogía de Fallot. En el Cateterismo se comprobó la existencia de un ductus con acentruada hinertensión pulmonar (presión sistémira) $v$ con un shunt. Aerecho-izquierdo predominante. (Fig. $\mathrm{N}^{2} 1$ ).

En esta enferma se intentó el cierre del ductus pero durante su oclusión transitoria se presentaba un marcado aumenio de la sobrecarga ventricular derecha y descenso casi inmediato a $O$ de la presión arterial periférica, por lo cual hubo de conservarse esta válvula de seguridad que representa el Ductus para estos enfermos una vez que se ha fijado la resistencia ar teriolar pulmonar.

El análisis de este caso nos permite valorar la importancia que tiene el diagnóstico precoz de este cuadro, como única posibilidad de efectuar una intervención correctora, ya que la mortalidad operato- ria sube a parejas con el grado de hipertensión llegado prácticamente a un $100 \%$ cuando la presión pulmonar se iguala a la sistémica y el shunt se hace predominantemente reverso. Con respecto a la indicación quirúrgica en esta etapa de la afección las opiniones aún subsisten divididas y hay cirujanos que preconizan la oclusión del ductus por etapas.

\section{RESUMEN}

Se analiza el cuadro clínico y hemodinámico de esta Cardiopatía en 9 enfermos entre las edades de 6 meses y 9 años con diagnóstico confirmado por medio del Cateterismo Cardíaco. Se recalca la importancia de este procedimiento auxiliar, dadas las dificultades diagnósticas que presenta esta afección especialmente en el lactante y niño pequeño.

Se insiste en la necesidad de un diagnóstico precoz como única forma de practicar una intervención oportuna y curativa.

Se dan a conocer los resultados del tratamiento.

\section{SUMMARY}

PATENT DUCTUS ARTERIOSUS WITH PULMONARY HYPERTENSION.

Clinical and hemodynamics studies in 9 children varying in age from 9 months to 9 years with patent ductus arteriosus with pulmonary hypertension are presented.

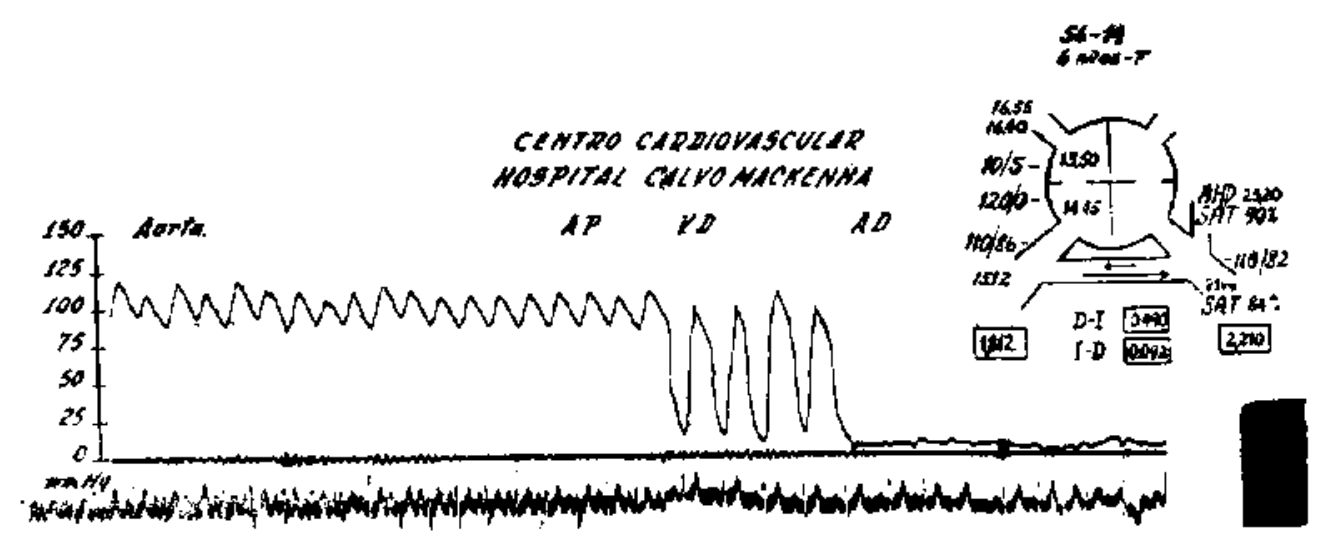

Figura N'1. Registro de la curva de presión de retirada desde la Aorta a Auricula derecha $y$ datos hemodinámicos. 
The importance of cardiac catherization in the diagnosis of this diseases, specially in small children and infants is stressed. They consider that an early diagnosis permits to obtain good results with the operation.

\section{BIHLIOGRAFÍA}

BOTHW HF., T. H.: VAN LINGEN, B.; WHDBORNE, I.; KAYE, J.; Me GREGOR, M; H.IIOT, G. A. Patent ductus arterlosus with portial reversal of shunt. A study of two cases. Am. Heart J., 44:360. 1052 .

BURCHEIL, B. B. - Vartationg in the clintcal and Pathologle pleture of patent ductus arterlosus Med. Clin. North America $32: 911$, 1948 .

BDRCHELL, H. B.; SWAN, H, J. C.; WOOD, E. F. Demostration of differentigl effects on pulmonary and systemle Arterial presiure by vartation in oxpgen content of inspired alr in patients with patent ductus arterlosus and pulmonary hypertenslon. Oliculation, 8:681-604 (Nov. 1953).

CABRER A. E.: MONROY J. R. - Systollo end Dastolle loading of the heart. II. Electrocardiographic data. deta. Am. Heart. J., $43: 609,1952$.

ChampMan, c. B.: RöBbins, \$, L. - Patent ductus arterloous with pulmonary vascular scierosis and cyanobis. Ann. Int. Med. $21: 312-323$. (Aur. 1944).

DAMAN. J F.: BURTAKIT, M. D.: EMWARDS. J. E.; DRY T. $\mathrm{J}$. PARKER, R. L - Bpstemic floht yentricle in patent ductur arterlosus: Report of a case with othstructive pulmonary vascular lestons. Proc. Staff Meet. Mayo Clln. 22:413-423. (Bept 1947).
DOUGLAS, J. M.; BURCHELL, M. D.; EDWARDS, J. E.; DRY, T. J.: PARKER, R, $\mathbf{L}$. Systemle rlght pentricles in patent ductus arterlosus: Report of a case with obstructive pulmonary rascular lesions. Froc. Staff Meat. Mayo Clln, 22:413-423 (Sep 1947).

DRY, T. J.; HARRINGTON, S. W.; EDWARDS, J. E. Irreversible carajac disease in adult life calused by delayed surgical closure of a patent ductus arterosus: Report of a case. Proc. Btalf Meet. Mayo Glin. 23:267, (June 1048)

DU SHANE, J. W. MONTGOMRRY, G. F. - Patent ductus arteriosus with pulmogary hypertension and stypjes] clinieal flndings. Proc. Staff Meet. Mayo cline, 23:505-506, 1948.

JOKNSON, 7 ; WBRNER. P.; KUSOHNER, M.; COJRNAND. A. - Intermittent retersal of now in a cBso of patent ductis arterlosus. A physiologle studg with autopsy findings. Clrculation 6:1293-1301 (June $1950)$.

NaDas, A. s. - Pediatric Cerdlology. Phlladelphla and London, $w$. B. Bannders Company. $195 \%$

NOVHLO. B.; LTMON. R.; BOURCHARD. F. - tJ nouvequ syudrome avec cyanose congentale: La pergistance du canal srterial avec hypertension pulmonalre. Communlcations. Ler, Congres Mondial de Cardlologie.

SHEPHFRD $\mathrm{J}$, $\mathrm{T}$; Demostration of pariations of aortic to pulmonary with a patent ductus grterlosus and pulmanary Rrtery flow during the cardlac crele in a patient hopertension. Froc. staff Meet. Mayo Clln. 29:301 308, 1954

SLVFR, A. W, KIRKIT, J, W. ELLLS, F, H. WOOD E. H. - Reoresion of pulmonary hypertension after closure of petent ductus arter10sils. Proc. Staff Meet. Mayo Clin, 29:293-309 (Mayo 1054)

SMTTK, G. - Patent ductus arterlosus with pulmonary hyoertension and reversed shunt. Brlt. Heart J. 16 232-240 (July 1954).

TADSSIG, F. B. - Congenital Malformations of the Heart, New York. Commonweelth Fund. 1947. 